\title{
A Variational Sum-Rule Approach to Collective Excitations of a Trapped Bose-Einstein Condensate
}

\author{
Takashi KimurA, Hiroki SAito and Masahito UedA \\ Department of Physical Electronics, Hiroshima University, Higashi-Hiroshima 739-8527, Japan \\ and Core Research for Evolutional Science and Technology (CREST), JST, Japan
}

(September 4, 2018)

\begin{abstract}
It is found that combining an excitation-energy sum rule with Fetter's trial wave function gives almost exact low-lying collective-mode frequencies of a trapped Bose-Einstein condensate at zero temperature.
\end{abstract}

Realizations of Bose-Einstein condensation (BEC) in trapped atomic gasest 1 t have enabled us to study many-body physics of weakly interacting bosons with unprecedented precision. Experiments on elementary excitations of BEd 58 present a new challenge because inhomogeneity and rotational symmetries of the system must be explicitly taken into account. A number of theoretical studies have been reported in literature 917 The results of numerical analyses 13 based on the Bogoliubov approximation 18,19 have so far found the best agreement with those of experiments. 目 Stringari国 obtained exact analytic expressions of collective-mode frequencies in the Thomas-Fermi (TF) limit.20 24 Pérez-García et al. 16 assumed a Gaussian trial wave function which yields correct collective-mode frequencies at the weak and strong coupling limits but overestimates them at an intermediate regime. In this Letter, it is found that combining an excitation-energy sum rule 25 with Fetter's trial wave function 26 gives the almost exact frequencies of a trapped Bose system at zero temperature. Our method will be shown to yield collective-mode frequencies in excellent agreement with experimental results as well as numerical ones based on the Bogoliubov approximation. 
Consider a Bose system described by the Hamiltonian, $H=T+U+H_{\text {int }}$, where $T=$ $-\left(\hbar^{2} / 2 M\right) \sum_{i} \nabla_{i}^{2}, U=(M / 2) \sum_{i}\left(\omega_{x}^{2} x_{i}^{2}+\omega_{y}^{2} y_{i}^{2}+\omega_{z}^{2} z_{i}^{2}\right)$ and $H_{\mathrm{int}}=\left(2 \pi \hbar^{2} a / M\right) \sum_{i \neq j} \delta\left(\mathbf{r}_{i}-\mathbf{r}_{j}\right)$ describe the kinetic energy, the confining potential energy and the inter-particle interaction energy, respectively. Here $M$ and $a$ denote the atomic mass and the $s$-wave scattering length, respectively, and $i$ denotes the particle index. Let $\{|n\rangle\}$ be a complete set of exact eigenstates of $H$ with eigenvalues $\left\{E_{n}\right\}$, where $n$ represents a complete set of quantum numbers that uniquely specify the many-body state of our system. For brevity of notation, we assume $n$ to be nonnegative integers such that $E_{0} \leq E_{1} \leq E_{2} \leq \cdots$. We are interested in low-lying excitations of many-body states which are excited by a general excitation operator $F$. Let $|0\rangle$ be the ground state and $|1\rangle$ be the lowest excited state with the excitation energy given by $\hbar \omega_{10}=E_{1}-E_{0}$. Because $\omega_{10}$ is not greater than $\hbar \omega_{n 0} \equiv E_{n}-E_{0}$ for $n \geq 2$, we obtain the following inequality

$$
\omega_{10}^{2} \leq \omega_{10}^{2} \frac{|\langle 1|F| 0\rangle|^{2}+\sum_{n \neq 1}|\langle n|F| 0\rangle|^{2}\left(\frac{\omega_{n 0}}{\omega_{10}}\right)^{3}}{|\langle 1|F| 0\rangle|^{2}+\sum_{n \neq 1}|\langle n|F| 0\rangle|^{2} \frac{\omega_{n 0}}{\omega_{10}}}
$$

which shows that an upper bound $\hbar \omega^{\text {upper }}$ of the lowest excitation energy is given by

$$
\hbar \omega^{\text {upper }}=\sqrt{m_{3} / m_{1}}
$$

where $m_{p} \equiv \sum_{n}|\langle 0|F| n\rangle|^{2}\left(\hbar \omega_{n 0}\right)^{p}$ is the $p$-th moment of the excitation energy. 15 , 25 Similar methods which evaluate the excitation energy have been used extensively in the field of nuclear physics.27 The advantage of this formula is that $m_{1}$ and $m_{3}$ can be expressed as expectation values of commutators between $F$ and $H$ with respect to the ground state $|0\rangle$ as $m_{1}=\frac{1}{2}\left\langle 0\left|\left[F^{\dagger},[H, F]\right]\right| 0\right\rangle$ and $m_{3}=\frac{1}{2}\left\langle 0\left|\left[\left[F^{\dagger}, H\right],[H,[H, F]]\right]\right| 0\right\rangle$, and we can therefore find $\hbar \omega^{\text {upper }}$ without the necessity of finding excited states. 15 To obtain $\omega^{\text {upper }}$ which is very close to $\omega_{10}$, we need to find a correct excitation operator $F$ and an accurate ground-state wave function.

We first consider the case of an axially symmetric trap $\left(\omega_{x}=\omega_{y} \equiv \omega_{\perp}\right)$, which is relevant to recent experiments.58 Fetter proposed a trial wave function for the condensate with repulsive interaction as 26 


$$
\Psi(\mathbf{r})=c_{0}\left(1-\frac{r_{\perp}^{2}}{d_{\perp}^{2} R_{\perp}^{2}}-\frac{z^{2}}{d_{z}^{2} R_{z}^{2}}\right)^{(1+\eta) / 2}
$$

if $\frac{r_{\perp}^{2}}{d_{\perp}^{2} R_{\perp}^{2}}+\frac{z^{2}}{d_{z}^{2} R_{z}^{2}} \leq 1$ and zero otherwise, where $r_{\perp} \equiv \sqrt{x^{2}+y^{2}}, c_{0}$ is the normalization constant, $d_{j} \equiv \sqrt{\hbar / M \omega_{j}}(j=\perp, z)$ is the oscillator length, and $R_{j}$ and $\eta$ are the variational parameters which are determined so as to minimize the total energy $E_{0}=\langle T\rangle+\langle U\rangle+\left\langle H_{\text {int }}\right\rangle$. Fetter showed that the trial wave function (3) smoothly interpolates between the noninteracting $\operatorname{limit}\left(\Psi(\mathbf{r}) \propto \exp \left(-r_{\perp}^{2} / 2 d_{\perp}^{2}-z_{\perp}^{2} / 2 d_{z}^{2}\right)\right.$ for $\left.\eta \sim \sqrt{R_{\perp}}, \sqrt{R_{z}} \rightarrow \infty\right)$ and the strongly interacting limit $\left(\Psi(\mathbf{r}) \sim c_{0}\left(1-r_{\perp}^{2} / d_{\perp}^{2} R_{\perp}^{2}-z^{2} / d_{z}^{2} R_{z}^{2}\right)^{1 / 2}\right.$ for $\left.\eta \rightarrow 0\right)$. However, how well it describes an intermediate regime had not been investigated until now. We therefore examine the accuracy of Fetter's variational wave function for various atomic numbers $N_{0}$. Table I compares the expectation values of the total, kinetic, potential, and interaction energies obtained by Fetter's wave function with those obtained numerically according to the method reported in ref. 28. The agreement is excellent and we thus conclude that eq. (3) serves our purpose very well.

Experimentally, low-lying collective modes are excited by superimposing small ac currents with appropriate phase relationship through magnetic coils. This leads to small modulations of the frequencies of the confining potential. We consider $|m|=2$ and $m=0$ modes which are relevant to recent experiments.5 The collective mode with magnetic quantum number $|m|=2$ describes the situation in which the condensate expands in one direction and simultaneously contracts in the other, thus maintaining its volume. This mode can be excited by modulating two radial trap frequencies out of phase but by the same amount $\delta \omega \ll \omega_{\perp}$. The resulting perturbation which defines the excitation operator is given by $F_{|m|=2}=M / 2 \sum_{i}\left\{\left[\left(\omega_{\perp}+\delta \omega\right)^{2}-\omega_{\perp}^{2}\right] x_{i}^{2}+\left[\left(\omega_{\perp}-\delta \omega\right)^{2}-\omega_{\perp}^{2}\right] y_{i}^{2}\right\} \approx M \omega_{\perp} \delta \omega \sum_{i}\left(x_{i}^{2}-y_{i}^{2}\right)$. We note that $F$ is proportional to $\sum_{i} r_{i}^{2}\left(Y_{2,2}+Y_{2,-2}\right)$, where $Y_{l m}$ is the spherical harmonic function.15 We can therefore take the excitation operator as $F_{|m|=2}=\sum_{i}\left(x_{i}^{2}-y_{i}^{2}\right)$, where the numerical factors are dropped because they are canceled in forming the ratio (2). The commutator $\left[H, F_{|m|=2}\right]$ is calculated as 


$$
\begin{aligned}
{\left[H, F_{|m|=2}\right] } & =\frac{1}{2 M} \sum_{i}\left[p_{x i}^{2}+p_{y i}^{2}, x_{i}^{2}-y_{i}^{2}\right] \\
& =-\frac{2 i \hbar}{M} \sum_{i}\left(x_{i} p_{x i}-y_{i} p_{y i}\right) .
\end{aligned}
$$

We obtain the first moment $m_{1}$ as

$$
\begin{aligned}
m_{1} & =\frac{1}{2}\left\langle\left[F_{|m|=2}^{\dagger},\left[H, F_{|m|=2}\right]\right]\right\rangle \\
& =\frac{2 \hbar^{2}}{M}\left\langle\sum_{i}\left(x_{i}^{2}+y_{i}^{2}\right)\right\rangle=\frac{8 \hbar^{2}}{M^{2} \omega_{\perp}^{2}}\left\langle U_{\perp}\right\rangle,
\end{aligned}
$$

where $U_{\perp}$ is a radial $(x$ or $y$ ) component of the trap potential energy and $\langle\cdots\rangle$ denotes the expectation value over the trial wave function (3). We calculate the third moment $m_{3}$ as

$$
\begin{aligned}
m_{3} & =\frac{1}{2}\left\langle\left[\left[F_{|m|=2}^{\dagger}, H\right],\left[H,\left[H, F_{|m|=2}\right]\right]\right]\right\rangle \\
& =\frac{16 \hbar^{4}}{M^{2}}\left(\left\langle T_{\perp}\right\rangle+\left\langle U_{\perp}\right\rangle\right),
\end{aligned}
$$

where $T_{\perp}$ is a radial ( $x$ or $y$ ) component of the kinetic energy. Because the upper-bound frequency does not directly depend on the interaction energy $\left\langle H_{\text {int }}\right\rangle$, we obtain the upperbound frequency as

$$
\omega^{\text {upper }}(m=2)=\omega_{\perp} \sqrt{2\left(1+\left\langle T_{\perp}\right\rangle /\left\langle U_{\perp}\right\rangle\right)} .
$$

In the absence of inter-particle interaction we find that $\left\langle T_{\perp}\right\rangle=\left\langle U_{\perp}\right\rangle$, so that $\omega^{\text {upper }}(m=$ 2) $=2 \omega_{\perp}$, while in the TF limit we have $\left\langle T_{\perp}\right\rangle=0$, so that eq. (7) reduces to the exact result $\omega^{\text {upper }}(m=2)=\sqrt{2} \omega_{\perp} .15$

The $m=0$ mode describes an excitation in which the condensate alternately expands and contracts in the radial direction. This part of the excitation is described by $F_{m=0}=$ $M / 2 \sum_{i}\left\{\left[\left(\omega_{\perp}+\delta \omega\right)^{2}-\omega_{\perp}^{2}\right] r_{\perp i}^{2}\right\} \approx M \omega_{\perp} \delta \omega \sum_{i} r_{\perp i}^{2} .15$ Because of the repulsive interaction, however, the condensate should also undergo oscillations in the axial direction which must be out of phase with the radial motion. form $F_{m=0}=M \omega_{\perp} \delta \omega \sum_{i}\left(r_{\perp i}^{2}-\alpha z_{i}^{2}\right)$, where $\alpha$ is another variational parameter. We note that $F$ is a linear combination of two modes $\sum_{i} r_{i}^{2}(n=1, l=m=0)$ and $\sum_{i} r_{i}^{2} Y_{2,0}\left(\theta_{i}, \phi_{i}\right)$ 
$(n=0, l=2, m=0)$. The excitation operator for the $m=0$ mode can therefore be taken as $F_{m=0}=\sum_{i}\left(x_{i}^{2}+y_{i}^{2}-\alpha z_{i}^{2}\right)$ for simplicity as in the $|m|=2$ case. The first moment $m_{1}$ is calculated as,

$$
m_{1}=\frac{4 \hbar^{2}}{M^{2}}\left(2 \frac{\left\langle U_{\perp}\right\rangle}{\omega_{\perp}^{2}}+\alpha^{2} \frac{\left\langle U_{z}\right\rangle}{\omega_{z}^{2}}\right)
$$

where $U_{z}$ is the axial component of the trap potential energy. The third moment $m_{3}$ is calculated as

$$
m_{3}=\frac{8 \hbar^{4}}{M^{2}}\left\langle\left[2\left(T_{\perp}+2 U_{\perp}\right)+\alpha^{2}\left(T_{z}+U_{z}\right)+\left(1-\frac{\alpha}{2}\right)^{2} E_{\mathrm{int}}\right]\right\rangle
$$

where $T_{z}$ is the axial component of the kinetic energy. We thus obtain the upper-bound frequency as

$$
\omega^{\text {upper }}(m=0, \alpha)=\left[2 \frac{2\left(\left\langle T_{\perp}\right\rangle+\left\langle U_{\perp}\right\rangle\right)+\alpha^{2}\left(\left\langle T_{z}\right\rangle+\left\langle U_{z}\right\rangle\right)+(1-\alpha / 2)^{2}\left\langle H_{\text {int }}\right\rangle}{2\left\langle U_{\perp}\right\rangle / \omega_{\perp}^{2}+\alpha^{2}\left\langle U_{z}\right\rangle / \omega_{z}^{2}}\right]^{\frac{1}{2}} .
$$

By minimizing $\omega^{\text {upper }}(m=0, \alpha)$ with respect to $\alpha$, we find $\omega^{\text {upper }}(m=0)=2 \omega_{\perp}$ in the noninteracting limit and $\omega^{\text {upper }}(m=0)=\omega_{\perp}\left(2+\frac{3}{2} \lambda^{2}-\frac{1}{2} \sqrt{9 \lambda^{4}-16 \lambda^{2}+16}\right)^{\frac{1}{2}}\left(\lambda \equiv \omega_{z} / \omega_{\perp}\right)$ in the strong interacting limit. This latter result is identical to that is obtained in ref.15 by another method. The agreement shows that the excitation operator $F_{m=0}$ that we chose is indeed correct. We also note that if $\omega^{\text {upper }}$ is maximized with respect to $\alpha$, we obtain $\omega^{\text {upper }}(m=0)=\omega_{\perp}\left(2+\frac{3}{2} \lambda^{2}+\frac{1}{2} \sqrt{9 \lambda^{4}-16 \lambda^{2}+16}\right)^{\frac{1}{2}}$, which also coincides with the result reported in ref. 15 with higher frequency. Because states excited by $F_{m=0}$ are restricted to states which are constructed by linear combinations of the $n=1, l=m=0$ mode and the $n=0, l=2, m=0$ mode, there should be two values of $\alpha$ that make $\omega^{\text {upper }}$ extremal and the corresponding states should describe the two lowest-energy excited states as obtained above.

Our method can also be applied to the dipole $(l=1)$ modes 15 , which correspond to the center-of-mass motion of the condensate, and should therefore not be affected by the inter-particle interaction. This is known as the generalized Kohn's theorem. We consider the excitation operator $F=M / 2 \sum_{i} \omega_{\perp}^{2}\left[\left(x_{i}+\delta\right)^{2}-x_{i}^{2}\right] \propto \sum_{i} x_{i} \propto \sum_{i} r_{i}\left(Y_{1,1}\left(\mathbf{r}_{i}\right)-Y_{1,-1}\left(\mathbf{r}_{i}\right)\right)$ or 
$F=M / 2 \sum_{i} \omega_{\perp}^{2}\left[\left(y_{i}+\delta\right)^{2}-y_{i}^{2}\right] \propto \sum_{i} r_{i}\left(Y_{1,1}\left(\mathbf{r}_{i}\right)+Y_{1,1}\left(\mathbf{r}_{i}\right)\right)$ for $|m|=1$, and $F=M / 2 \sum_{i} \omega_{z}^{2}\left[\left(z_{i}+\right.\right.$ $\left.\delta)^{2}-z_{i}^{2}\right] \propto \sum_{i} z_{i} \propto \sum_{i} r_{i} Y_{10}\left(\mathbf{r}_{i}\right)$ for $m=0$. Substituting $F$ into Eq. (22), we easily obtain $\omega^{\text {upper }}(l=1,|m|=1)=\omega_{\perp}$ and $\omega^{\text {upper }}(l=1, m=0)=\omega_{z}$. We thus find that the dipolemode frequencies obtained by our method coincide with trap frequencies, being independent of the strength of interaction.

Figure 1 compares our analytical results (solid curves) with the experimental data (dots) taken from ref. 5 for ${ }^{87} \mathrm{Rb}$ atoms, where we use the same parameters as in the experiment; $a=109 a_{0}$ ( $a_{0}$ is the Bohr radius) and $\omega_{z} / \sqrt{8}=\omega_{\perp}=2 \pi \times 132 \mathrm{~Hz}$. Our results for both $m=0$ and $|m|=2$ modes are in excellent agreement with those of the experiment and with those obtained with the Bogoliubov approximation ${ }^{13}$. We have also calculated the upper-bound frequencies using numerically calculated values of $\langle T\rangle$ and $\langle U\rangle$ for 4500 atoms and obtained $\omega^{\text {upper }}(|m|=2)=1.454 \omega_{\perp}$ and $\omega^{\text {upper }}(m=0)=1.871 \omega_{\perp}$. These results are in excellent agreement with those obtained using Fetter's variational wave function, that is, $\omega^{\text {upper }}(|m|=2)=1.450 \omega_{\perp}$ and $\omega^{\text {upper }}(m=0)=1.870 \omega_{\perp}$.

We briefly describe the results of our study for the case of a spherically symmetric trap $\left(\omega_{\perp}=\omega_{z} \equiv \omega_{0}\right)$, where we can compare our results with those reported in ref. 15 and with numerical ones based on the Bogoliubov approximation 17 . For the quadrupole mode $(l=2)$, we consider the case of $m=2$ without loss of generality, where $F=M \delta \omega \sum_{i}\left(x_{i}^{2}-y_{i}^{2}\right)$, and find that $\omega^{\text {upper }}(l=0)=\omega_{0} \sqrt{2+\langle T\rangle /\langle U\rangle}$. For the monopole mode $(n=1, l=0)$, where $F=M \delta \omega \sum_{i} r_{i}^{2}$, we find that $\omega^{\text {upper }}(l=0)=\omega_{0} \sqrt{5-\langle T\rangle /\langle U\rangle}$, where we have used the virial identity $2\langle T\rangle-2\langle U\rangle+3\left\langle H_{\text {int }}\right\rangle=0$. These results are the same as those reported in ref. 15, although analytic evaluation of $\langle T\rangle$ and $\langle U\rangle$ in an intermediate strength of interaction is not so far available from the TF limit.

In Fig. 2, we present our results for both modes with the same parameters $\left(\omega_{0}=\right.$ $\left.2 \pi \times 200 \mathrm{~Hz}, a=110 a_{0}\right)$ as those reported in ref. 17 using the Bogoliubov approximation. Our results are almost indistinguishable from those obtained numerically using the Bogoliubov approximation.

In conclusion, we have presented an analytical method to evaluate almost exactly the 
collective-mode frequencies of a trapped Bose gas. The results are in excellent agreement with those of the Bogoliubov approximation 13 and those of the experiments 5 at zero temperature. 


\section{REFERENCES}

${ }^{1}$ M.H. Anderson, J.R. Ensher, M.R. Matthews, C.E. Wieman and E.A. Cornell: Science 269 (1995) 198.

${ }^{2}$ C.C. Bradley, C.A. Sackett, J.J. Tollet and R.G. Hulet: Phys. Rev. Lett. 75 (1995) 1687.

${ }^{3}$ K.B. Davis, M.-O. Mewes, M.R. Andrews, N.J. van Druten, D.S. Durfee, D.M. Kurn and W. Ketterle: Phys. Rev. Lett. 75 (1995) 3969.

${ }^{4}$ C.C. Bradley, C.A. Sackett and R.G. Hulet: Phys. Rev. Lett. 78 (1997) 985.

${ }^{5}$ D.S. Jin, J.R. Ensher, M.R. Matthews, C.E. Wieman and E.A. Cornell: Phys. Rev. Lett. 77 (1996) 420.

${ }^{6}$ M.-O. Mewes, M.R. Andrews, N.J. van Druten, D.M. Kurn, D.S. Durfee, C.G. Townsend and W. Ketterle: Phys. Rev. Lett. 77 (1996) 988.

${ }^{7}$ D.S. Jin, M.R. Matthews, J.R. Ensher, C.E. Wieman and E.A. Cornell: Phys. Rev. Lett. 78 (1997) 764.

${ }^{8}$ D.M. Stamper-Kurn, H.-J. Miesner, S. Inouye, M.R. Andrews and W. Ketterle: Phys. Rev. Lett. 81 (1998) 500.

${ }^{9}$ A. Smerzi and S. Fantoni: Phys. Rev. Lett. 78 (1997) 3589.

${ }^{10}$ Y. Castin and R. Dum: Phys. Rev. Lett. 77 (1996) 5315.

${ }^{11}$ A.L. Fetter and D.L. Feder: Phys. Rev. A 58 (1998) 3185.

${ }^{12}$ Yu. Kagan, E.L. Surkov and G.V. Shlyapnikov: Phys. Rev. A 55 (1997) R18.

${ }^{13}$ M. Edwards, P.A. Ruprecht, K. Burnett, R.J. Dodd and C.W. Clark: Phys. Rev. Lett. 77 (1996) 1671.

${ }^{14}$ B.D. Esry: Phys. Rev. A 55 (1997) 1147.

${ }^{15}$ S. Stringari: Phys. Rev. Lett. 77 (1996) 2360. 
${ }^{16}$ V.M. Pérez-García, H. Michinel, J.I. Cirac, M. Lewenstein and P. Zoller: Phys. Rev. Lett. 77 (1996) 5320 .

${ }^{17}$ D.A.W. Hutchinson, E. Zaremba and A. Griffin: Phys. Rev. Lett. 78 (1997) 1842.

18 N.N. Bogoliubov: J. Phys. (U.S.S.R.) 11 (1947) 23;

${ }^{19}$ A.L. Fetter: Ann. Phys. (N.Y.) 70 (1972) 67.

${ }^{20}$ V.V. Goldman, I.F. Silvera and A.J. Leggett: Phys. Rev. B 24 (1981) 2870

${ }^{21}$ D.A. Huse and E.D. Siggia: Sov. Phys. JETP 13, 451 (1961).

${ }^{22}$ D.A. Huse and E.D. Siggia: J. Low Temp. Phys. 46 (1982) 137.

${ }^{23}$ E.P. Gross: Nuovo Cimento 20 (1961) 454.

${ }^{24}$ E.P. Gross: J. Math. Phys. 4 (1963) 195.

${ }^{25}$ H. Wagner: Z. Physik 195 (1966) 273.

${ }^{26}$ A.L. Fetter: J. Low Temp. Phys. 106 (1997) 643.

${ }^{27}$ O. Bohigas, A.M. Lane and J. Martorell: Phys. Rep. 52 (1979) 267.

${ }^{28}$ F. Dalfovo and S. Stringari: Phys. Rev. A 53 (1996) 2477. 


\section{TABLES}

TABLE I. Comparison of total energy $\left\langle E_{\text {tot }}\right\rangle$, kinetic energy $\langle T\rangle$, confining-potential energy $\langle U\rangle$ and interaction energy $\left\langle H_{\text {int }}\right\rangle$ obtained using Fetter's variational wave function with those obtained numerically according to ref. 28 (in parentheses) for an axially-symmetric trap. We take $\sqrt{8} \omega_{\perp}=\omega_{z}=2 \pi \times 220 \mathrm{~Hz}$ and $a=100 a_{0}$. Energies are shown in units of $\hbar \omega_{\perp}$ and $N_{0}$ is the atom number.

\begin{tabular}{ccccc}
\hline \hline$N_{0}$ & $\left\langle E_{\text {tot }}\right\rangle$ & $\langle T\rangle$ & $\langle U\rangle$ & $\left\langle H_{\text {int }}\right\rangle$ \\
\hline 1000 & $3.86(3.84)$ & $0.75(0.76)$ & $2.17(2.15)$ & $0.95(0.93)$ \\
10000 & $7.83(7.76)$ & $0.46(0.45)$ & $4.61(4.57)$ & $2.76(2.74)$ \\
20000 & $10.06(9.98)$ & $0.40(0.38)$ & $5.95(5.91)$ & $3.70(3.68)$ \\
\hline \hline
\end{tabular}




\section{FIGURES}

FIG. 1. Collective-mode frequencies of the $|m|=2$ and the $m=0$ modes in an axially symmetric trap, where we assume $\omega_{z} / \sqrt{8}=\omega_{\perp}=2 \pi \times 132 \mathrm{~Hz}$ and $a=109 a_{0}$. The solid curves show our results, the dots show the experimental data reported in ref. 5, and the dashed lines show the results in ref. 15 .

FIG. 2. Collective-mode frequencies for the quadrupole and the monopole modes in a spherically symmetric trap. The dashed lines show the results reported in ref. 17 . 


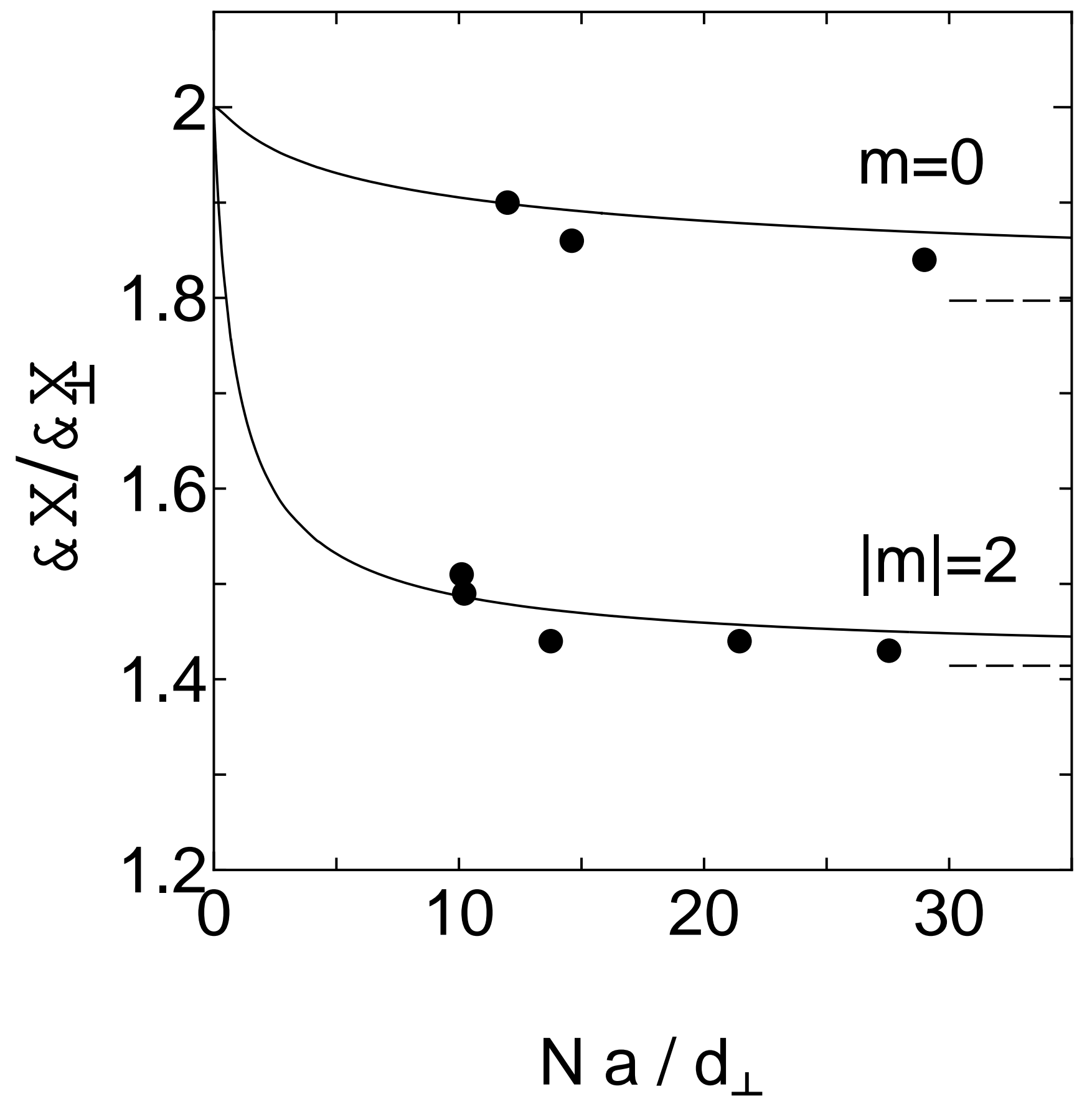

Figure 1 


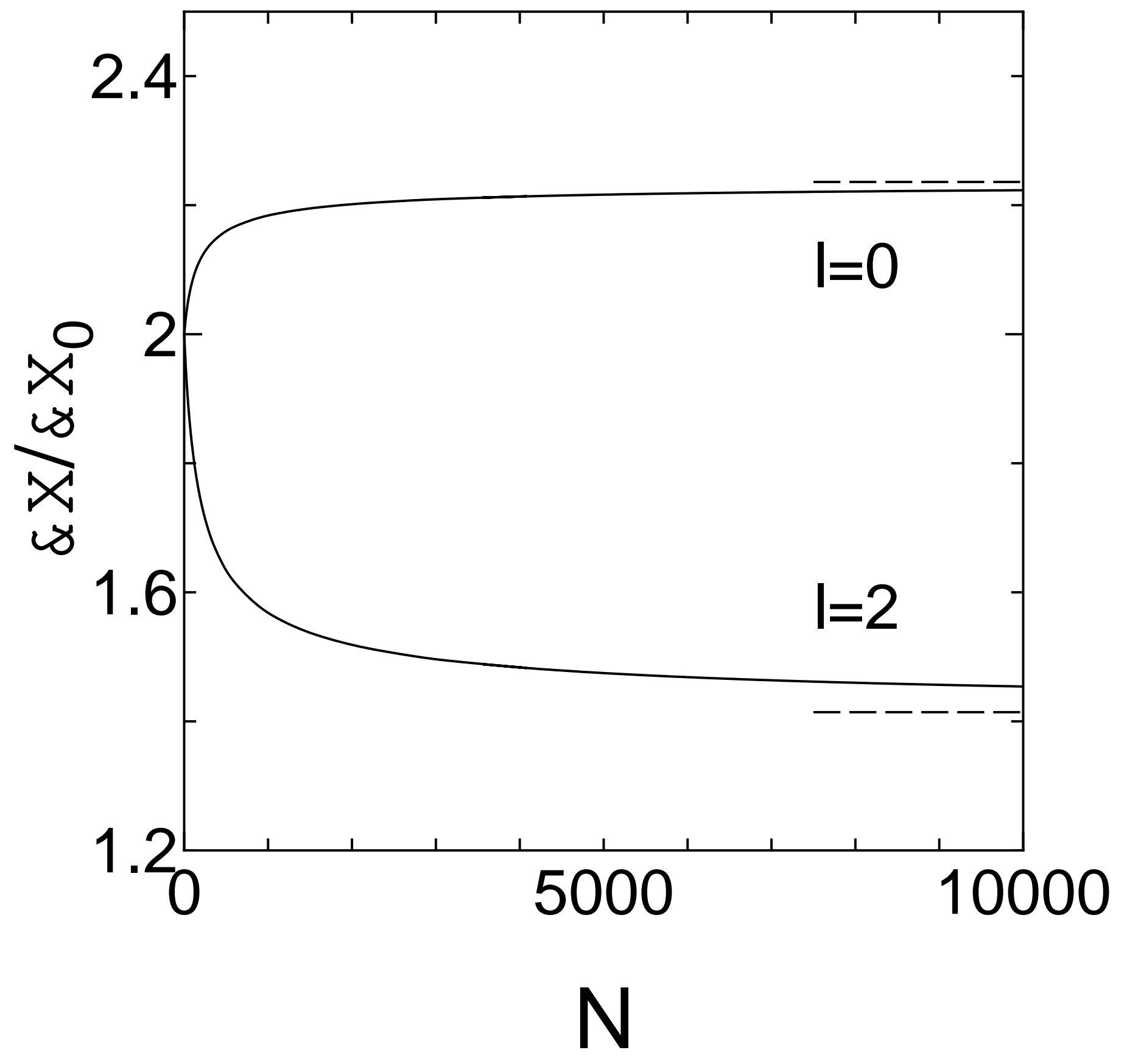

Figure 2 